\title{
X-ray rotational modulation of a supersaturated star in IC 2391
}

\author{
A. Marino ${ }^{1}$, G. Micela ${ }^{2}$, G. Peres ${ }^{1}$, and S. Sciortino ${ }^{2}$ \\ 1 Dipartimento di Scienze Fisiche e Astronomiche, Sez. di Astronomia, Università di Palermo, \\ Piazza del Parlamento 1, 90134 Palermo, Italy \\ 2 INAF - Osservatorio Astronomico di Palermo G.S. Vaiana, Piazza del Parlamento 1, 90134 Palermo, Italy
}

Received 7 April 2003 / Accepted 8 July 2003

\begin{abstract}
We present evidence of X-ray rotational modulation on VXR45, a young fast rotator star, member of IC 2391. It is a dG9 spectral type star whose rotational period and X-ray luminosity make it a supersaturated star. Our X-ray observation, made with EPIC/PN on XMM-Newton, covers about two photometric rotational periods. The detection of X-ray rotational modulation implies the presence of structural inhomogeneities. Possible interpretations are presented and discussed.
\end{abstract}

Key words. open clusters and associations - stars: individual: IC 2391 - stars: coronae - stars: late-type - stars: rotation $\mathrm{X}$-ray: stars

\section{Introduction}

Rotational modulation by a factor of two, due to the nonuniform distribution of emitting regions, is observed in solar coronal emission at any phase of the solar cycle. Since we cannot resolve stellar coronal structures, rotational modulation is one of the main methods to find evidence of non-uniform distribution of emitting stellar coronal regions and to investigate geometrical and physical properties of stellar coronal structures. Therefore, detection of X-ray rotational modulation on a very active star is particularly important to gain some insight into the physical mechanisms which operate on these stars. Observational data have shown that the most active stars, rotating above $\sim 15-20 \mathrm{~km} \mathrm{~s}^{-1}$, reach a maximum X-ray luminosity such that $L_{\mathrm{x}} / L_{\mathrm{bol}}$ saturates at $\sim 10^{-3}$, where $L_{\mathrm{bol}}$ indicates the star's bolometric luminosity (e.g. Vilhu 1984). Saturation lacks a clear interpretation: it could be the effect of dynamo saturation, or rather could correspond to the total filling of the star's surface by active regions, as originally suggested by Vilhu (1984). Furthermore ROSAT observations have shown that very fast rotators $\left(v \sin i>100 \mathrm{~km} \mathrm{~s}^{-1}\right)$ exhibit a level of X-ray luminosity a factor of 3-5 below the saturated level (Randich 1998). The origin of this phenomenon, named supersaturation by Prosser et al. (1996) is still unexplained and several hypotheses have been formulated. Supersaturation could be the result of an overall decrease of dynamo efficiency at very high rotation rate or the consequence of the redistribution of the radiative output due to the lower stellar gravity because of the enhanced rotation (Randich 1998). A different explanation is

Send offprint requests to: A. Marino, e-mail: marino@astropa.unipa.it that very rapid rotation could lead to a higher coronal temperature and to the shift of the X-ray emission out of the ROSAT passband (Randich 1998). Stępień et al. (2001) suggested a common explanation for the supersaturation both in ultra fast rotators stars (UFRs) and in W UMa binaries via a decreased coverage of the stellar surface by X-ray emitting regions, although they noted that the physical mechanisms leading to such a decreased coverage can be different for the two star classes. For UFRs they proposed strong polar updrafts within a convection zone, driven by nonuniform heating from below. Thanks to the high sensitivity and broad bandpass of XMM-Newton, we can now test some of these hypotheses. However presently there is no consensus on phenomena occurring in a stellar corona either in the saturated or in supersaturated regime.

There have been several attempts to observe rotational modulation of stellar coronal emission in fast rotating stars (e.g. Collier Cameron et al. 1988; Vilhu 1992; Agrawal \& Vaidya 1988; Kürster et al. 1992; Drake et al. 1994; Guedel et al. 1995; Kürster et al. 1997; Audard et al. 2001; García-Alvarez et al. 2003 ) in order to find a possible signature of non-uniform distribution of emitting coronal regions. In most cases the results were affected by frequent superimposed flares or poor sampling over more than one rotation period (e.g. Collier Cameron et al. 1988; Vilhu 1992). Probably one of the best examples of X-ray rotational modulation is that reported by Guedel et al. (1995): $\mathrm{X}$-ray flux variations within a factor of two in the ROSAT AllSky Survey light-curve of EK Draconis, a dG0 star with an optical rotation period of 2.7-2.8 day, just slightly too long for the star to be in the saturation regime. Guedel et al. (1995) found that the X-ray light curve is significantly variable in the ROSAT energy band with the emission from the cooler plasma being 
responsible for the rotational modulation but with the hot plasma approximately constant. On the other hand Kürster et al. (1997) find only a partial evidence of X-ray rotational modulation of the X-ray flux implying structural inhomogeneities in the saturated star AB Dor. Similar results are found in BeppoSAX observations of $\mathrm{AB}$ Dor by Franciosini et al. (2002). On the contrary Guedel et al. (2001) find no evidence for X-ray rotational modulation in a XMM-Newton observation of AB Dor. Analogously also Singh et al. (1999) find no indication of $\mathrm{X}$-ray rotational modulation both in the saturated fast rotators HD197890, of similar spectral type of VXR45, and in G1890, observed with ASCA.

In this Letter we present the unambiguous detection of $\mathrm{X}$-ray rotational modulation in a fast rotating star member of IC 2391: VXR45, observed with XMM-Newton/EPIC/PN. VXR45 is a dG9 star with a very short photometric period of 0.223 days (Patten \& Simon 1996), and $v \sin i>$ $200 \mathrm{~km} \mathrm{~s}^{-1}$ (Stauffer et al. 1997). Its $\log L_{\mathrm{x}}$, as measured with ROSAT, ranges between 30.12 and $30.10 \mathrm{erg} / \mathrm{s}$, but the range of $\log \left(L_{\mathrm{x}} / L_{\mathrm{bol}}\right)$ between -3.60 and -3.62 puts the star below the saturation level, making it a supersaturated star.

\section{The observation and data analysis}

The observation analyzed here was dedicated to the young open cluster IC 2391 as part of EPIC/PN GTO time and it has been performed in November 20, 2001. Here we present EPIC/PN data relative to the star VXR45, located at RA $(\mathrm{J} 2000)=$ $8^{\mathrm{h}} 42^{\mathrm{m}} 14.8^{\mathrm{s}}$, Dec $(\mathrm{J} 2000)=-52^{\circ} 56^{\prime} 01^{\prime \prime}$; it has $V=10.70$ and $B-V=0.81$.

The nominal duration of the observation is $50 \mathrm{ks}$; after the selection of the good time intervals (i.e. those of adequately low background emission) we retained $\sim 33 \mathrm{ks}$. The X-ray data reduction was performed with the $S A S 5.4 .1$ software $^{1}$. The star is one of the brightest in the field and it is located 3.96' off-axis, far from the EPIC/PN CCD gaps.

\subsection{Light-curve}

In order to determine the count rate, we extracted photons from a circular region centered on the source and selected so as to include $\sim 80 \%$ of the source photons (Ghizzardi 2001; Saxon 2002), corresponding to a radius of $37^{\prime \prime}$. The total number of photon counts in the $0.3-7.8 \mathrm{keV}$ band amounts to 7431 ; the local background has been determined from a source-free region near VXR45 for a total of 506 counts. Figure 1 shows the light-curves of VXR45 obtained in the $0.3-7.8 \mathrm{keV}$ band (top) and of the corresponding background (bottom). The X-ray modulation, with a period very similar to the photometric one $(0.223$ days $=19.3 \mathrm{ksec})$, is clearly evident in Fig. 1 . Note that other short term variability not due to $\mathrm{X}$-ray rotational modulation may also be present in the light-curve but the data clearly show that the variability observed cannot be explained with individual large flares.

In order to confirm that modulation of the X-ray emission is due to rotation we have folded the X-ray light-curve with

\footnotetext{
1 Available at http://xmm.vilspa.esa.es/sas
}

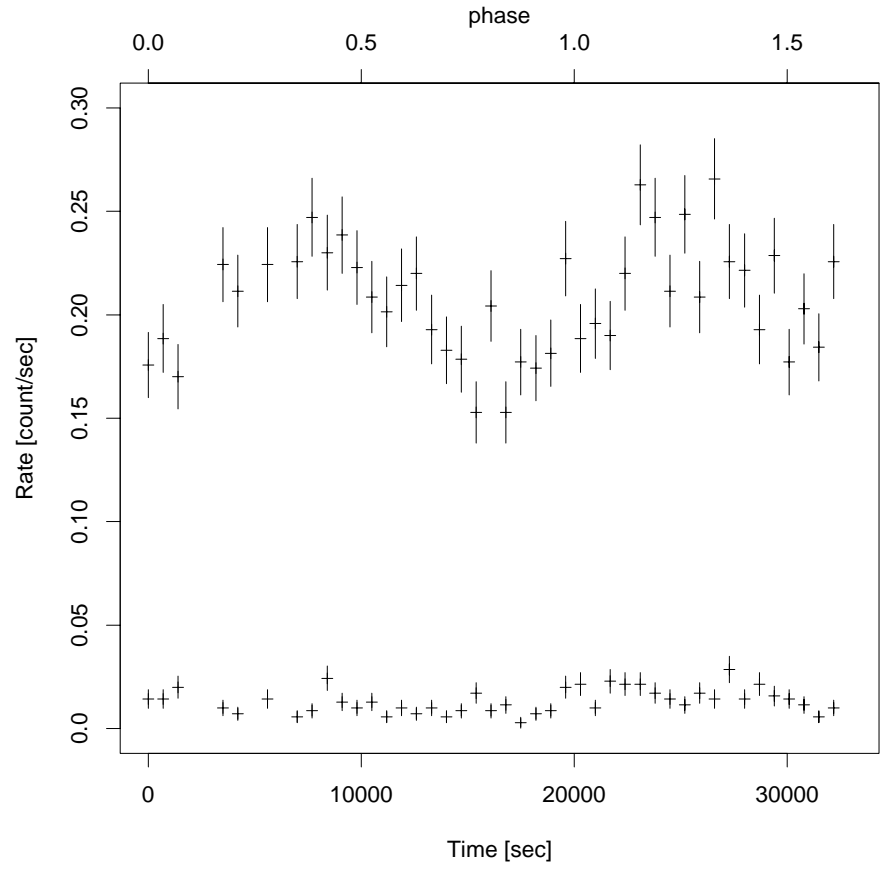

Fig. 1. X-ray light-curve of VXR45 (top) and background (bottom) as seen with EPIC/PN in the $0.3-7.8 \mathrm{keV}$ band, time bins are $700 \mathrm{~s}$ long. The photometric rotational period of $\sim 19.3 \mathrm{ksec}$ is well visible.

the photometric rotational period as shown in Fig. 2. Phaserelated variability is clearly evident; the $\mathrm{X}$-ray variations in the light-curve are $\sim 30 \%$ of the average count rate. Using a radius of $0.9 R_{\odot}$ adequate for the star's age and spectral type (Siess et al. 2000) and knowing $P_{\text {rot }}$ and $v \sin i$, we obtain an inclination angle close to $90^{\circ}$. This makes the star an ideal target to detect rotational modulation due to a longitudinal structure. In order to explore if differences exist between modulation of cool and hot plasma as in EK Dra (Guedel et al. 1995) we show in Fig. 3 the ratio between the hard count rate $(1.5-3.5 \mathrm{keV})$ and the soft count rate $(0.3-1.5 \mathrm{keV})$ versus the time ${ }^{2}$. There is no strong evidence of spectral changes in the soft and hard passbands, and the $\chi^{2}$ test applied to the hard/soft count-rate ratio confirms that the variations observed are those expected in a random sample. We tried different energy boundaries in soft and hard bands definition and the result does not change. The lack of modulation in the hard $(1.5-3.5 \mathrm{keV}) /$ soft $(0.3-1.5 \mathrm{keV})$ ratio is consistent with the hypothesis that hotter and cooler plasmas are co-spatial. On the contrary the X-ray rotational modulation of EK Dra (Guedel et al. 1995) was detected predominantly in the soft ROSAT band $(0.1-0.4 \mathrm{keV})$, that in practice is outside the XMM-Newton bandpass, while it was approximately constant in the hard ROSAT band $(0.4-2 \mathrm{keV})$.

2 The break point $1.5 \mathrm{keV}$ separates the line-dominated Fe $\mathrm{L}$ shell $+\mathrm{NeIX} / \mathrm{X}+\mathrm{MgXI} / \mathrm{XII}$ region from the part dominated by bremsstrahlung continuum. Hence the adopted ratio maps on temperature. 


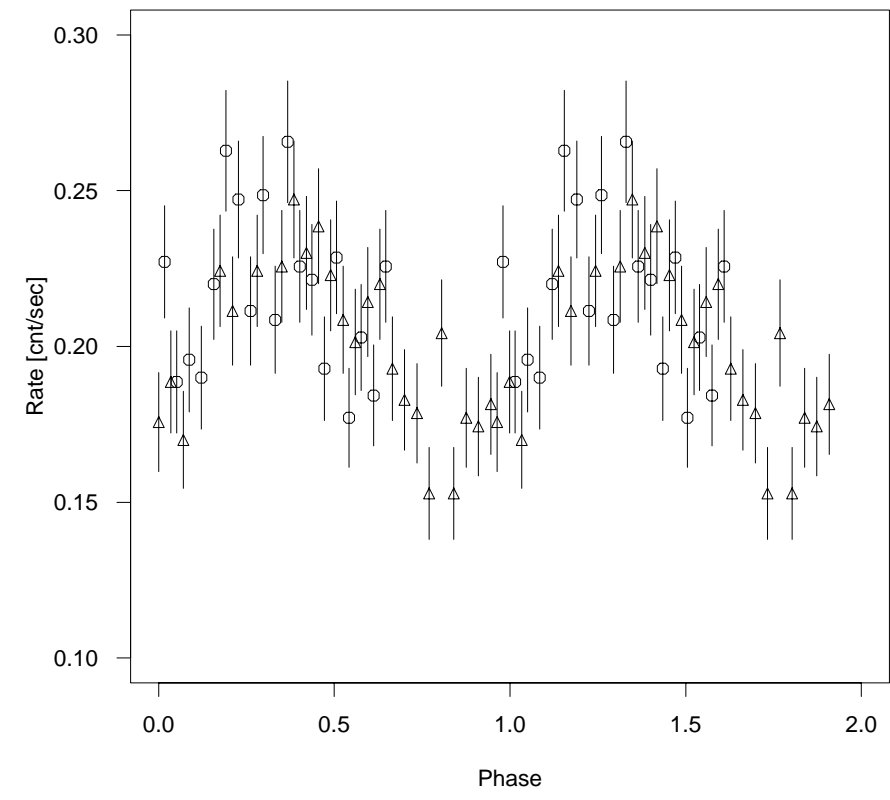

Fig. 2. X-ray data of Fig. 1 folded with the rotational period vs. phase. Circles are points observed at $t<0.223$ days (the photometric period) since the observation start and triangles those observed at $t>0.223$ days. For clarity we report two photometric periods.

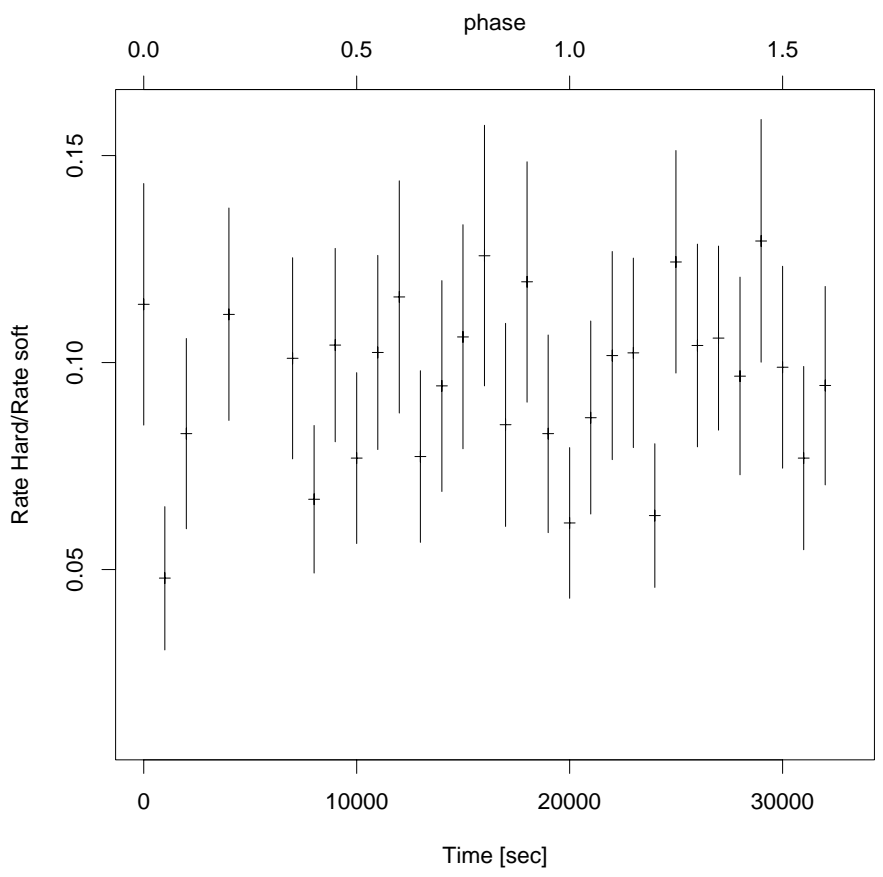

Fig. 3. Ratio of hard count rate $(1.5-3.5 \mathrm{keV})$ to soft count rate $(0.3-1.5 \mathrm{keV})$ vs. time for VXR45, time bins are $1000 \mathrm{sec}$ long. There is no evidence for modulation of the ratio.

\subsection{Count rate, flux and luminosity}

We extracted the spectrum of the single and doubly flagged PN events with energy in $0.3-3.5 \mathrm{keV}$ band that contains the essential part of the source (Fig. 4). We found that a 2-T APEC spectral model provides the best fit to the data, as measured with a $\chi^{2}$ test $\left(\chi_{v}^{2}=1.01\right.$ for 189 degrees of freedom). The $N_{\mathrm{H}}$ value was fixed to $10^{19.5} \mathrm{~cm}^{-2}$ (Patten \& Simon 1996). The two temperatures in the model, as well as the corresponding

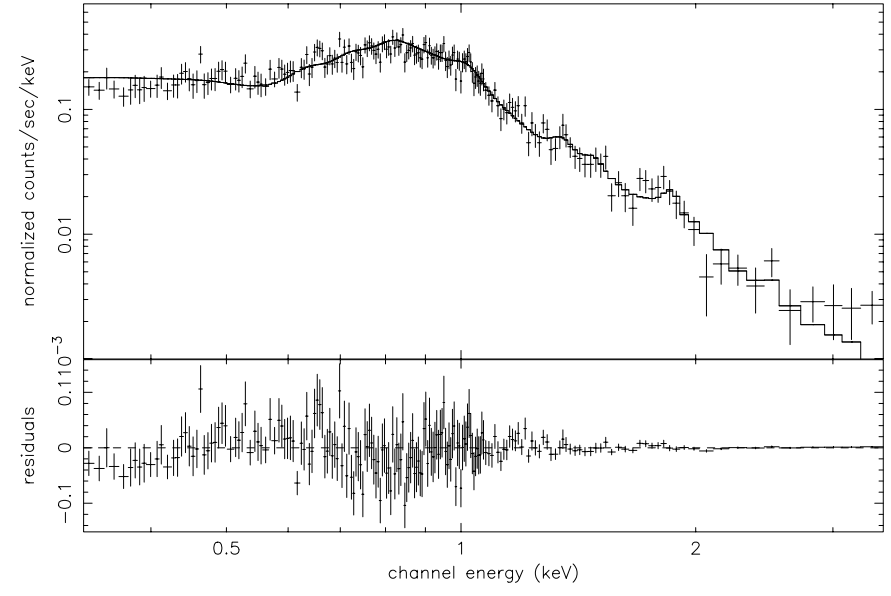

Fig. 4. X-ray spectrum of VXR45 with the best fit model superimposed.

emission measures and the abundance $Z$ were allowed to vary as free parameters in the fitting process. We obtained temperatures of $0.61 \mathrm{keV}$ and $1.19 \mathrm{keV}$, with a ratio of emission measure of $E M_{\text {cold }} / E M_{\text {hot }} \approx 1.42$, and $Z \sim 0.27 Z_{\odot}$. The spectral fit yielded an $X$-ray flux, in the $0.3-3.5 \mathrm{keV}$ band, of $4.77 \times 10^{-13} \mathrm{ergs} \mathrm{cm}^{-2} \mathrm{~s}^{-1}$ corresponding to an average $\mathrm{X}$-ray luminosity of $1.50 \times 10^{30} \mathrm{erg} / \mathrm{s}$ adopting a distance of $162 \mathrm{pc}$ (Patten \& Simon 1996) and $\log \left(L_{\mathrm{x}} / L_{\mathrm{bol}}\right)=-3.54$. Assuming that the spectrum does not change, as suggested by Fig. 3, the peak to peak variations of $\log \left(L_{\mathrm{x}}\right)$ are 30.04-30.28 and $\log \left(L_{\mathrm{x}} / L_{\text {bol }}\right)$ ranges between -3.44 and -3.68 . These intervals include the values observed with ROSAT.

\section{Discussion and conclusions}

Notwithstanding the wealth of available data, X-ray rotational modulation is very hard to detect in active stars, as discussed in the Introduction. We observed VXR45 a fast rotator member of IC 2391, with XMM-Newton/EPIC/PN for $~ 33 \mathrm{ks}$, corresponding to $\sim 2$ photometric rotational periods. We find unambiguous evidence of $\mathrm{X}$-ray rotational modulation from a supersaturated star with a level of X-ray emission 100 times larger than the solar one. We note that we detect X-ray rotational modulation in the $0.3-7.8 \mathrm{keV}$ energy band while the X-ray rotational modulation of EK Dra (Guedel et al. 1995), was detected in the softer and smaller ROSAT energy band (0.1-0.4 keV); furthermore VXR45 is a dG9 supersaturated star located on the left of the $\log L_{\mathrm{x}} / L_{\text {bol }}$ vs. $P_{\text {rot }}$ plot (being a very fast rotator) (Randich 1998) while EK Dra is a dG0 with a similar $\log \left(L_{\mathrm{x}} / L_{\mathrm{bol}}\right)=-3.53$ but a rotation period 10 times slower that puts it on the right side of the same plot and just before the saturated regime ${ }^{3}$. On the other hand X-ray rotational modulation has been detected in at least some observations in AB Dor, a star in the saturated regime, while it has not been detected in other saturated stars (HD 197890, Gl890).

VXR45 was extensively observed with ROSAT, but an analysis of the PSPC observations of those IC 2391 cluster

\footnotetext{
${ }^{3} L_{\mathrm{x}} / L_{\mathrm{bol}}$ has been obtained taking $L_{\mathrm{x}}=10^{29.92} \mathrm{erg} / \mathrm{s}$ (Guedel et al. $1995)$ and $L_{\mathrm{bol}}=2.70 \times 10^{33} \mathrm{erg} / \mathrm{s}$ computed using the transformations by Flower (1996).
} 
members whose photometric rotational period was known (including VXR45), failed to detect X-ray rotational modulation in any of the X-ray light-curves (Patten \& Simon 1996). These authors note that the segments of their observations were not optimally spaced for this kind of analysis. Furthermore, they studied the time variations of the PSPC VXR45 lightcurve, with several statistical tests but obtaining contradicting results.

The new XMM-Newton observation allows us to detect $\mathrm{X}$-ray rotational modulation thanks to the quasi-continuous time coverage, spanning twice the photometric period. The detection of X-ray rotational modulation, implying the presence of non-uniformly distributed active regions on the star, is in some sense surprising, since bright coronae variations are typically due to flares.

Thanks to the broad energy bandpass of XMM-Newton, we can exclude the hypothesis that this supersaturated star has higher coronal temperature that may cause most of the $\mathrm{X}$-ray emission to be outside the ROSAT passband. The detection of X-ray rotational modulation indicates that the star is not completely covered by active regions. Furthermore, as shown in Fig. 3 there is no strong evidence for spectral variations in the soft and hard passbands. This is consistent with the hypothesis that the modulation we observe is mainly due to a longitudinal concentration of X-ray emitting material, and that at all times the emission is largely due to the same mixture of emitting structures. The average surface X-ray flux, derived from the $\mathrm{X}$-ray luminosity divided by the stellar surface is $3.0 \times 10^{7} \mathrm{erg} \mathrm{s}^{-1} \mathrm{~cm}^{-2}$ and ranges between $3.9 \times 10^{7}$ and $2.2 \times 10^{7} \mathrm{erg} \mathrm{s}^{-1} \mathrm{~cm}^{-2}$. These surface X-ray fluxes values are a bit higher than those of solar active regions (Orlando et al. 2001) but intermediate between active regions and solar flares (Reale et al. 2001), and the X-ray temperatures are also intermediate between solar active regions and the flares.

Our finding of a incomplete coverage of supersaturated stellar surface are qualitatively in agreement with the explanation proposed by Stępień et al. (2001). A possible scenario is that a large polar region is always present and another region at lower latitude is responsible for the observed modulation, albeit other scenarios are possible. Observations of other saturated and supersaturated stars will help in clarifying this picture.
Acknowledgements. We acknowledge financial support from ASI and MIUR. We wish to thank the referee M. Guedel for useful comments.

\section{References}

Agrawal, P. C., \& Vaidya, J. 1988, MNRAS, 235, 239

Audard, M., Güdel, M., \& Mewe, R. 2001, A\&A, 365, L318

Collier Cameron, A., Bedford, D. K., Rucinski, S. M., Vilhu, O., \& White, N. E. 1988, MNRAS, 231, 131

Drake, J. J., Brown, A., Patterer, R. J., et al. 1994, ApJ, 421, L43

Flower, P. J. 1996, ApJ, 469, 355

Franciosini, E., Pallavicini, R., Maggio, A., Cutispoto, G., \& Tagliaferri, G. 2002, in 1st Potsdam Thinkshop on Sunspots and Starspots; Poster Proceedings of the Meeting, Potsdam, Federal Germany, 53

García-Alvarez, D., Foing, B. H., Montes, D., \& et al. 2003, A\&A, 397, 285

Ghizzardi, S. 2001, EPIC-MCT-TN-011, Tech. rep., EPIC Milano Calibration Team

Guedel, M., Audard, M., Briggs, K., et al. 2001, A\&A, 365, L336

Guedel, M., Schmitt, J. H. M. M., Benz, A. O., \& Elias, N. M. 1995, A\&A, 301, 201

Kürster, M., Schmitt, J. H. M. M., Cutispoto, G., \& Dennerl, K. 1997, A\&A, 320, 831

Kürster, M., Schmitt, J. H. M. M., \& Fleming, T. A. 1992, in Cool Stars, Stellar Systems, and the Sun, ASP Conf. Ser., 26, 109

Orlando, S., Peres, G., \& Reale, F. 2001, ApJ, 560, 499

Patten, B. M., \& Simon, T. 1996, ApJS, 106, 489

Prosser, C. F., Randich, S., Stauffer, J. R., Schmitt, J. H. M. M., \& Simon, T. 1996, AJ, 112, 1570

Randich, S. 1998, in Cool Stars, Stellar Systems, and the Sun, ASP Conf. Ser., 154, 501

Reale, F., Peres, G., \& Orlando, S. 2001, ApJ, 557, 906

Saxon, R. D. 2002, XMM-CCF-REL-116, Tech. rep., XMM-SOC

Siess, L., Dufour, E., \& Forestini, M. 2000, A\&A, 358, 593

Singh, K. P., Drake, S. A., Gotthelf, E. V., \& White, N. E. 1999, ApJ, 512,874

Stauffer, J. R., Hartmann, L. W., Prosser, C. F., et al. 1997, ApJ, 479, 776

Stępień, K., Schmitt, J. H. M. M., \& Voges, W. 2001, A\&A, 370, 157

Vilhu, O. 1984, A\&A, 133, 117

Vilhu, O. 1992, in Evolutionary Processes in Interacting Binary Stars, IAU Symp., 151, 61 